\title{
Encuesta BIARE 2012: Análisis del bienestar subjetivo de los mexicanos y modelamiento estructural
}

\author{
BIARE survey 2012: subjective analysis related to mexican \\ people well-being and structural modeling
}

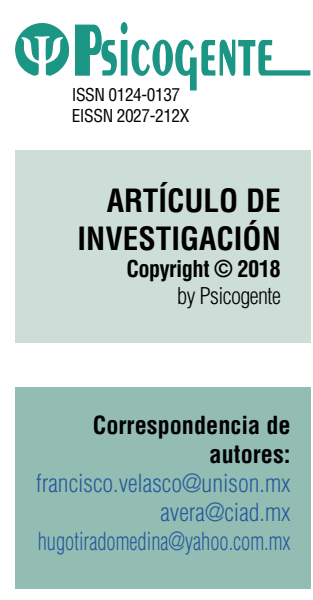

Recibido: $30-01-17$ Aceptado: $18-06-18$ Publicado: 08-01-19

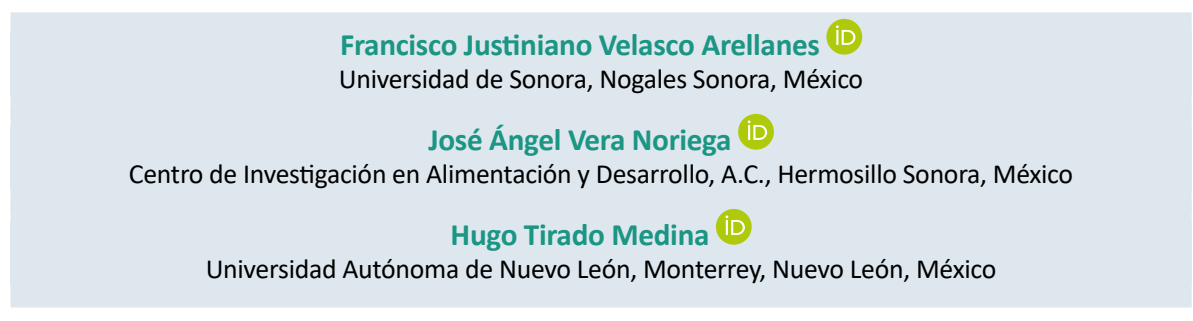

Resumen

Objetivo: El bienestar de una sociedad es un constructo considerado como multidimensional (componentes objetivos y subjetivos). La encuesta nacional de Bienestar Autorreportado (BIARE) se diseñó para evaluar el bienestar de la población mexicana. No obstante, no se ha explorado la estructura y relación que tienen los módulos (sub-escalas) para explicar el bienestar de los mexicanos. Así, el objetivo del estudio es explorar la estructura y relación que tienen los módulos en la encuesta BIARE, 2012 (Instituto Nacional de Estadística y Geografía [INEGI], 2012) mediante modelamiento estructural.

Método: Se realizó análisis de datos secundarios de la base BIARE, la cual es aleatoria y representativa a nivel nacional. Participaron 10.654 ciudadanos con un rango de edad entre 18 y 70 años; 5.967 eran mujeres y 4.687 hombres.

Resultados: Se encontraron tres modelos. En un primer modelo se concibió la variable latente Bienestar general asociada con variables empíricas Solvencia, Felicidad, Satisfacción y Autodeterminación. En un segundo modelo se proponen dos variables latentes: 1) Bienestar percibido, asociada a tres variables empíricas; a) Satisfacción con la vida, b) Autodeterminación y c) Felicidad; 2) Socio-cultura, relacionada también con tres variables empíricas: a) Altruismo, b) Cultivado y c) Relaciones virtuales. Un tercer modelo de trayectorias para variables latentes mostró ajustes satisfactorios que explican relaciones entre la variable Felicidad con Solvencia, Satisfacción y Autodeterminación, consecutivamente.

Conclusiones: La información que se puede obtener del cuestionario BIARE es relevante para evaluar la percepción de los ciudadanos y el entorno inmediato, como la familia. Pero no permite una evaluación precisa sobre la contribución de los factores meso y macro-sociales en el bienestar de los mexicanos. Es necesario que BIARE integre un modelo multidimensional de bienestar.

Palabras Clave: Bienestar, modelos estructurales, factores psicosociales, calidad de vida.

\section{Abstract}

Objective: The National Self-Reported Well-being Survey (BIARE) was designed to assess well-being in mexican population, considering well-being of a society as a multidimensional construct (objective and subjective components). However, the structure and relationship between modules (sub-scales) to explain mexican well-being has not been explored yet. Thus, this study aims to explore the structure and relationship among those sub-scales based on the BIARE survey 2012 (INEGI, 2012) through structural modeling.

Method: BIARE, Secondary data analysis, which is random and representative of Mexican people was conducted; 10.654 citizens, who were between 18 and 70 years were sampled; 5.967 women and 4.687 men.

Results: As a result, three models were found. In a first model, the latent variable related to general well-being associated with empirical variables such as: solvency, happiness, satisfaction and self-determination were

Cómo citar este artículo (APA):

Velasco Arellanes, F. J., Vera Noriega, J. A. y Tirado Medina, H. (2019). Encuesta BIARE, 2012: Análisis del bienestar subjetivo de los mexicanos y

modelamiento estructural. Psicogente 22(41), 1-20. https://doi.org/10.17081/psico.22.41.3357 
considered. In a second model, two latent variables are proposed: 1) Perceived well-being, associated with three empirical variables as follows: a) life Satisfaction b) Self-determination and c) Happiness; 2) Socioculture, also related to three empirical variables, which are: a) Selflessness, b) Cultivated relationships and c) Virtual relationships. A third model of trajectories for latent variables showed adjustments that explain relations among variable Happiness with Solvency, Satisfaction and Self-determination.

Conclusions: The information that can be obtained from BIARE questionnaire is relevant to evaluate citizens's perception and immediate environment, like family. But it does not allow for an accurate assessment about contribution of meso and macro-social factors on mexican's well-being. It is necessary that BIARE integrate a multidimensional model of well-being.

Keywords: Well-being, structural modeling, psychosocial factors, quality of life.

\section{INTRODUCCIÓN}

El interés por evaluar de manera científica las condiciones que producen satisfacción a los individuos surgió en la década de 1930 bajo enfoques estrictamente económicos, los cuales se caracterizaron por una filosofía económica tradicional que imputó la condición de bienestar desde los criterios de los expertos y desestimó la atribución de valor que las personas realizan sobre sus condiciones de vida (Rojas \& Jiménez, 2008). El término bienestar bajo el modelo estrictamente económico hace referencia a la posesión o al tener; entre los estimadores estadísticos que se utilizan están: el Producto Interno Bruto (PIB), el nivel de alfabetismo de una población, las tasas de mortalidad infantil, el ingreso per cápita, la esperanza de vida, los indicadores de estabilidad política, el consumo de energía por país, el promedio del tamaño de las viviendas, el número de personas que las habitan y los niveles de las libertades civiles (Van Praag \& Frijters, 1999).

En los años setenta y ochenta se criticó la visión económica de bienestar, se aludió a que los indicadores económicos no eran suficientes para medir las condiciones de vida de las personas, ya que su percepción de bienestar no necesariamente se relaciona con el tener, ni con la mejora de las condiciones económicas generales de los países (Cardona \& Agudelo, 2005).

A partir de la filosofía económica han surgido varios enfoques económicos con los que se analiza el bienestar de las poblaciones, pero uno de los más importantes es llamado "la escuela Leyden". Esta asumió por primera vez la importancia de incorporar los juicios de las personas al análisis científico del bienestar (Van Praag, 1968; 1971). El enfoque, o Escuela Leyden al inicio se centró en la evaluación del ingreso, posteriormente el interés se extendió, se consideró la utilidad del ingreso y la satisfacción del ingreso o, por decirlo de otra manera, en la satisfacción económica que reportaban las personas (Van Praag \& Frijters, 1999). 
Gracias a la acertada contribución que hizo la escuela Leyden con la incorporación del componente subjetivo al análisis del bienestar, el concepto de utilidad siguió permaneciendo en el trasfondo de su filosofía sugiriendo que las personas establecen sus condiciones de bienestar mediante la maximización o la búsqueda de la mayor cantidad de bienes que provee el medioambiente. Este resabio filosófico que acompañó a esta aproximación económica generó críticas en el sentido de que se argumentó que los individuos no usan estimadores de probabilidad, que las personas no son sistemas de cómputo que se comportan estimando fríamente el costo y las ganancias que ofrece el medioambiente. La paradoja conocida como Allais, se opone empíricamente al concepto de utilidad, e indica que los individuos no usan probabilidades porque subestiman las pequeñas y desestiman las altas (Allais \& Hagen, 1979).

Así también, las aportaciones que han realizado Kahneman y sus colaboradores al análisis económico ha demostrado que los componentes afectivos están implicados de manera importante en nuestras decisiones y que "la fría racionalidad" que se atribuye a "un cerebro que estima sus ganancias", bajo el concepto de utilidad no tiene un fundamento teórico que pueda explicar el comportamiento humano en términos de la búsqueda del bienestar por razones estrictamente económicas. Es decir, Kahneman encontró que las personas no utilizan estimaciones de probabilidad, recordando que la teoría de la probabilidad es el conjunto de regularidades que deben ser satisfechas para asignar números (véase Kahneman, Knetsch \& Thaler, 1991; Kahneman \& Tversky, 1979; Kahneman, Wakker \& Sarin, 1997).

Actualmente el análisis del bienestar implica necesariamente la evaluación del componente subjetivo de las personas. Esta última vertiente se centra en los componentes subjetivos de los individuos y de manera indirecta de los componentes económicos; los representantes de esta corriente asumen que los indicadores importantes para evaluar bienestar son las respuestas verbales que emiten los sujetos en relación a sus condiciones de vida. Además, se ha demostrado ampliamente que el reporte de las personas y de sus condiciones de vida definen su bienestar; esta última perspectiva no ha carecido de críticas por el amplio espectro de conceptos que se tiene en la noción de calidad de vida, para algunos es felicidad (Veenhoven, 1996), el promedio de la satisfacción (Dow \& Juster, 1985), un balance homeostático entre lo que el ambiente provee y el individuo percibe del mismo (Cummins, 2005) y bienestar subjetivo (Diener, 2000). 
Recientemente el enfoque de medir el progreso de las sociedades surgió en un consenso internacional; como resultado de esta reunión se emitió un reporte de la Comisión sobre la Medición del Desempeño Económico y el Progreso Social (Stiglitz, Sen \& Fitoussi, 2010). Posterior a este consenso internacional, en México se realizó la Conferencia Latinoamericana para la Medición del Bienestar y la Promoción del Progreso de las Sociedades. Fue en ese contexto que a través del Instituto Nacional de Estadística y Geografía (INEGI) se inició la medición del Bienestar Subjetivo mediante el diseño de un módulo de preguntas que sería integrado a la Encuesta Nacional de Gastos de los Hogares (Instituto Nacional de Estadística y Geografía [INEGI], 2012).

La encuesta BIARE aplicada en el año 2012 en México se concibió bajo un enfoque multidimensional, en la que las condiciones de vida y las percepciones sobre el contexto personal y social de los individuos es importante para explicar la dinámica del bienestar. Esta medida está validada y confiabilizada por la Organización para la Cooperación y el Desarrollo Económico (OCDE), (Hoorn, 2007). No obstante, el potencial estadístico que tiene la encuesta BIARE para inferir las condiciones de bienestar de los mexicanos y de que ya se cuenta con datos más actualizados, los módulos y sus componentes, no se han sometido a prueba mediante modelamiento. Por esta razón, el objetivo del presente estudio es someter a prueba mediante modelación estadística estructural la encuesta o módulo BIARE aplicada en el año 2012 (Instituto Nacional de Estadística y Geografía [INEGI], 2012) para cuantificar sus relaciones y comparar resultados.

\section{MÉTODO}

\subsection{Diseño metodológico}

El estudio obedece a un enfoque asociativo, no experimental, de carácter retrospectivo, de tipo transversal, con un procedimiento de muestreo aleatorio estratificado proporcional (McMillan y Schumacher, 2008, p.19). Dadas estas características resulta un diseño de investigación útil para la generación de objetivos de tipo explicativo.

\subsection{Tamaño y características de la muestra}

Para el objetivo propuesto se utilizó la base de datos derivado del estudio Ilevado a cabo por INEGI (2012), el cual fue retrospectivo de tipo transversal, aleatorio y representativo a nivel nacional. Está compuesto por una población de 10.654 ciudadanos mexicanos (con posibilidad de ejercer el voto), de los cuales 5.967 son mujeres y 4.687 son hombres. El rango de edades es de 18 
a 70 años predominando la población joven con una media de 39,52. El 56,3 $\%$ de los ciudadanos se encuentra en edades entre 18 y 40 años. El 17,5 \% vive en unión libre, 6,9 \% es separado, 2,9 \% divorciado, 4,4 \% viudo, 22,5\% soltero y el $46,2 \%$ casado.

\subsection{Instrumento de medición}

\subsubsection{Encuesta nacional de Bienestar Autorreportado}

En México se realizó la Conferencia Latinoamericana para la Medición del Bienestar y la Promoción del Progreso de las Sociedades, la cual se desarrolló en mayo de 2011 en la Ciudad de México. Fue en ese contexto que la autoridad del gobierno federal mexicano encargó a los directivos del Instituto Nacional de Estadística y Geografía (INEGI) que iniciaran la primera medición de Bienestar Subjetivo mediante el diseño de un módulo de preguntas que sería integrado a la Encuesta Nacional de Gastos de los Hogares (INEGI, 2012).

BIARE es una encuesta o módulo de preguntas que tiene como objetivo conocer la satisfacción con la vida, la felicidad y el balance afectivo de los ciudadanos de México (adultos con capacidad de voto). Es una encuesta que se consideró como experimental o de pilotaje dado que en el año 2012 era la primera vez que se recogían datos de esta naturaleza con una muestra representativa y avalada por una institución oficial del gobierno mexicano.

Es así como se propuso el módulo experimental BIARE, el cual se diseñó como una encuesta de autollenado, pero se realizaron variantes de excepción para que fuesen apoyadas aquellas personas que tuviesen problemas de lectoescritura, visuales o que solicitaran expresamente que la encuesta fuese leída por el encuestador.

Para el diseño de la encuesta BIARE se contactó a expertos que siguieron las recomendaciones de la OCDE para medir bienestar subjetivo, satisfacción con la vida y balance afectivo. Así también se consideraron los contenidos de la encuesta Social Europea para medir la incorporación de los individuos a su entorno social; esta última se incorporó para obtener información sobre derechos humanos, cohesión y participación ciudadana, familia, género y vulnerabilidad, salud física y mental.

El formato de autollenado BIARE tiene una tasa de no respuesta $17 \%$ mayor en comparación a La Encuesta Nacional de Gastos de los Hogares (ENGASTO), por esta razón se hizo un ajuste de los ponderadores para compensar sesgos por la no respuesta, con lo cual se recuperaron 10.654 cuestionarios 
como válidos. La encuesta BIARE se aplicó en el primer trimestre de 2012 (enero-marzo).

\subsection{Procedimiento}

La base de datos se obtuvo de la página del INEGI de la encuesta aplicada en el primer trimestre de 2012. La experiencia y sistematicidad que ha logrado este instituto, como institución oficial del estado mexicano que realiza evaluaciones estadísticas en los diversos rubros y actividades del país, garantiza que la encuesta BIARE sea confiable en términos del tamaño de muestra y la metodología de levantamiento. Por esta razón se utilizó la muestra total de 10.654 cuestionarios ajustados por ponderaciones por el INEGI (2012). Posteriormente se hizo análisis de estadística descriptiva con SPSS para detectar los valores extremos o casos de anomalía estadística, el cual indicó que los 10.654 casos eran viables para realizar análisis estadísticos. La encuesta tiene dos partes, en una primera los contenidos son: satisfacción con la vida en general ( 2 reactivos), satisfacción con los dominios específicos (14), eudaimonia (11), estados afectivos (10) y plenitud (7). La segunda parte de la encuesta tiene contenidos sobre la caracterización y contextualización del individuo. Incluye, características sociodemográficas (4), eventos y situaciones recientes (57), vida social, participación y crecimiento personal (45) y aspectos biográficos (15).

Con el fin de poder evaluar la relación que guardan los diferentes contenidos de la encuesta BIARE (módulo) se realizaron cambios a los valores de captura originales con la finalidad de que tuvieran un sentido positivo. Debido a los múltiples niveles de medida de los ítems o reactivos de la encuesta (dicotómico, ordinal y continuo) se agruparon las preguntas que estaban distribuidas en diferentes lugares de la escala general pero que pertenecían al mismo constructo y así crear un puntaje total (sumatoria). Por ejemplo, las preguntas: ENCSAT, Satis_1-11, Aspect_1-4 (son etiquetas originales de las preguntas que miden variables relacionadas con "Satisfacción"), formaron un constructo llamado Satisfacción. La falta de homogeneidad de los reactivos impidió el uso de Análisis factorial. Otro cambio consistió en agrupar algunas preguntas para formar nuevos contenidos, por ejemplo, autodeterminación, se constituye por la sumatoria de los reactivos; logros, "decis", "fuerte" y "depende" (las cuales son etiquetas originales en la base original de la base de datos BIARE). Este puntaje total refleja las creencias de las personas en relación con la importancia que han tenido sus propias decisiones en su vida. 
Bajo los lineamientos anteriores se identificaron 14 indicadores con puntajes en forma de sumatoria que podían ser relacionados con la variable latente "bienestar subjetivo", los cuales fueron nombrados: 1) Felicidad, 2) Satisfacción, 3) Autodeterminación, 4) Altruismo, 5) Ecológico, 6) Cultivado, 7) Participación Social, 8) Relaciones Virtuales, 9) Relaciones Directas, 10) Solvencia, 11) Políglota, 12) Adversidad, 13) Sufre Violencia y 14) Apoyo Percibido. Creadas las sumatorias se aplicó estadísticas descriptivas y análisis multivariado. A continuación, se presenta la Tabla 1, donde se describen los reactivos que componen las sumatorias y el tipo de reactivo (escalar o dicotómico).

Tabla 1.

Sumatorias, escalas y tipos de reactivos que fueron considerados para el análisis

\begin{tabular}{|c|c|c|c|}
\hline SUMATORIA & REACTIVOS & \multicolumn{2}{|c|}{ TIPO DE REACTIVO } \\
\hline Felicidad & Gr_Feliz, feliz, alegria 1-3, Ed_Anim 1-2 & $\begin{array}{l}\text { Gr_Feliz escala } \\
\text { Feliz escala } \\
\text { Alegria dicotómico } \\
\text { Ed_Anim escala }\end{array}$ & $\begin{array}{r}1-4 \\
0-10 \\
1-2 \\
0-10\end{array}$ \\
\hline Satisfacción & ENCSAT, Satis_1-11, Aspect_1-4 & Escala & $0-10$ \\
\hline Autodeterminación & Logros, decis, fuerte y depende & $\begin{array}{l}\text { Logros dicotómica } \\
\text { Decis dicotómica } \\
\text { Fuerte escala } \\
\text { Decis escala }\end{array}$ & $\begin{array}{r}1-2 \\
1-2 \\
0-10 \\
0-10\end{array}$ \\
\hline Altruismo & Apoyo_1-5 & Dicotómico & $1-2$ \\
\hline Ecología & Ecología_1-10. & Dicotómico & $1-2$ \\
\hline Cultivado & SEMP_1-16 & Dicotómico & $1-2$ \\
\hline Participación Social & Perten_1-12 & Dicotómico & $1-2$ \\
\hline Relaciones Virtuales & contac_1 al 2, correo_1 al 1 + redsoc & Dicotómico & $1-2$ \\
\hline Relaciones Directas & Reune_A y Reune_f & Escala & $0-7$ \\
\hline Solvencia & Tarjeta, presta_1-6, avión, viajo, compper, internet). & Dicotómico & $1-2$ \\
\hline Poliglota & LENGUA_1-3 & Dicotómica & $1-2$ \\
\hline Adversidad & $\begin{array}{c}\text { dificult, Ayuda, dific_f, Padece, cercano_1-2, per_pad1-3, } \\
\text { padgrav_1-5, padacc_1-5. }\end{array}$ & Dicotómica & $1-2$ \\
\hline Violencia & maltra_1-12; agres_1-3; amena_1-3 & Dicotómica & $1-2$ \\
\hline Apoyo percibido & familia, no_fam, vecino & Escala & $0-7$ \\
\hline
\end{tabular}

Nota: Para una mejor identificación y descripción completa de los reactivos (véase el listado de variables 2012).

\subsection{Tipo de Análisis}

Para el análisis de los datos de la base BIARE se usaron diferentes estadísticos. Para analizar las variables individuales se utilizaron estadísticos descriptivos, tanto de tendencia central como de dispersión. Para las relaciones generales entre variables se usó el estadístico coeficiente de correlación producto-momento de Pearson. Para las diferencias entre grupos se utilizó el estadístico ANOVA y el estadístico para detectar diferencias específicas entre los grupos 
la prueba post hoc Bonferroni. Para demostrar relaciones de regresión entre las variables se usó el estadístico análisis de trayectorias. Para la determinación de la presencia de un constructo latente que representará a los indicadores se utilizó el análisis factorial confirmatorio (AFC). Los paquetes estadísticos utilizados fueron el SPSS 22 y el AMOS.

\subsection{Conflicto de interés}

Los autores no tienen conflicto de intereses asociados al financiamiento o acceso a las bases de datos.

\section{RESULTADOS}

En los primeros resultados, Tabla 2, se presentan los estadísticos descriptivos (media, desviación estándar, mínimos, máximos y percentiles) de las escalas que se obtuvieron de la suma de reactivos. En los resultados de dichos estadísticos podemos apreciar una distribución sesgada, por ejemplo, en Felicidad y Satisfacción las personas presentan altos niveles de respuesta. Asimismo, las personas reportan sentirse felices (50\% arriba de la media) y satisfechos ( $56 \%$ arriba de la media) mientras que en Adversidad se encontró que casi el $60 \%$ responde con niveles por debajo de la media. En cuanto a la escala Violencia el $70 \%$ respondió por debajo de la media.

Tabla 2.

Estadística Descriptiva de las dimensiones diseñadas para el estudio

\begin{tabular}{|c|c|c|c|c|c|}
\hline & & NIVEL DE INSTRUCCIÓN & SATISFACCIÓN & ADVERSIDAD & AUTODETERMINACIÓN \\
\hline \multicolumn{2}{|c|}{ Media } & 3,67 & 118,62 & 20,55 & 20,27 \\
\hline \multicolumn{2}{|c|}{ DE } & 1,83 & 22,27 & 1,38 & 3,30 \\
\hline \multicolumn{2}{|c|}{ Mínimo } & 0 & 0 & 17 & 2 \\
\hline \multicolumn{2}{|c|}{ Máximo } & 7 & 160 & 35 & 24 \\
\hline \multirow{6}{*}{ Percentiles } & 25 & & & & \\
\hline & 50 & 2,0 & 106,0 & 20,0 & 19,0 \\
\hline & 75 & & & & \\
\hline & & 4,0 & 122,0 & 20,0 & 21,0 \\
\hline & & 5,0 & 134,0 & 21,0 & 23,0 \\
\hline & & POLÍGLOTA & SOLVENCIA & ALTRUISMO & ECOLÓGICO \\
\hline \multicolumn{2}{|c|}{ Media } & 5,72 & 18,76 & 6,37 & 15,09 \\
\hline \multicolumn{2}{|c|}{$\mathrm{DE}$} & 0,509 & 2,15 & 1,30 & 2,40 \\
\hline \multicolumn{2}{|c|}{ Mínimo } & 3 & 11 & 5 & 10 \\
\hline \multicolumn{2}{|c|}{ Máximo } & 6 & 27 & 10 & 20 \\
\hline \multirow{5}{*}{ Percentiles } & 25 & & & & \\
\hline & 50 & 5,0 & 18,0 & 5,0 & 14,0 \\
\hline & 75 & & & & \\
\hline & & 6,0 & 19,0 & 6,0 & 15,0 \\
\hline & & 6,0 & 20,0 & 7,0 & 17,0 \\
\hline
\end{tabular}




\begin{tabular}{|c|c|c|c|c|}
\hline & SUFRE DE VIOLENCIA & CULTIVADO & DECESOS & FELICIDAD \\
\hline Media & 18,60 & 20,27 & 0,85 & 41,89 \\
\hline$D E$ & 1,26 & 2,78 & 0,62 & 7,72 \\
\hline Mínimo & 12 & 16 & 0 & 5 \\
\hline Máximo & 32 & 32 & 8 & 52 \\
\hline $\begin{array}{ll} & 25 \\
\text { Percentiles } & 50 \\
& 75\end{array}$ & 18,0 & 18,0 & 0,00 & \\
\hline
\end{tabular}

Se realizó análisis de correlación entre las escalas encontrándose que la correlación más alta fue de $r=0,66$ entre Felicidad y Satisfacción con la vida. La correlación menor con $r=0,10$ ocurrió entre las escalas Ecológico y Relaciones virtuales. En general las escalas no presentan colinealidad; es decir, ninguna de ellas es resultante de la combinación de otras.

Se realizó un primer análisis comparando mujeres versus hombres encontrándose diferencias en casi todas las escalas, no obstante, la distancia aritmética entre las medias es insignificante, esto es debido a que las muestras grandes afectan positivamente el valor de Alfa. Por ejemplo, al comparar hombres y mujeres se encontraron diferencias significativas en la escala Felicidad con un valor de $p=0,000$, pero la distancia aritmética entre las medias es muy cercana. Los hombres obtuvieron una media de felicidad de 42,12 mientras que las mujeres de 41,70. Así pues, estos resultados, aun cuando son estadísticamente significativos, pueden no tener relevancia en términos psicológicos.

Con el conocimiento de los resultados anteriores se dividió la muestra en tres grupos en relación al constructo Felicidad (33 \% por grupo); se utilizó el estadístico ANOVA (Bonferroni) para comparar los tres grupos definidos por felicidad con las variables Dormir mal y Satisfacción encontrándose diferencias significativas entre las medias $F(2 \mathrm{gl})=617,49 ; \mathrm{p}=0,000$ y $\mathrm{F}(2 \mathrm{gl})=2797,93$, $p=0,000$, respectivamente. Esta prueba indicó que el grupo 1 , bajos puntajes de felicidad, tiene puntajes mayores en dormir mal $(2,50, D E=0,933)$, mientras que el grupo 2, puntajes medios en felicidad, tiene puntajes menores en dormir mal $(M=2,09 ; D E=0,836)$. En esta lógica el grupo 3 presentó mayores diferencias $(M=1,17 ; D E=0,838)$. La prueba ANOVA proporcionó información similar cuando los tres grupos definidos por felicidad definidos como variable independiente en relación a la variable Satisfacción, el grupo 1 de menor felicidad obtuvo la media más pequeña en Satisfacción ( $M=100,45, D E=22,8)$, el grupo 2 (intermedio en felicidad) obtuvo una media un poco mayor 
$(M=119,3 ; D E=15,2)$ y finalmente el grupo 3 de felicidad obtuvo la media con mayor puntaje en satisfacción ( $M=132,6 ; \mathrm{DE}=22,7)$.

\subsection{Análisis Factorial Confirmatorio}

Las primeras tentativas sobre la búsqueda de modelos que ordenaran los diferentes factores o constructos en la base BIARE dejó evidente la existencia de dos modelos separados, uno de ellos relacionado al Bienestar compuesto por las variables Satisfacción, Autodeterminación, Solvencia y Felicidad; el otro compuesto por Altruismo, Ecológico, Cultivado y Relaciones Virtuales, este último factor recibió el nombre de Sociocultural.

EI AMOS buscó integrar un constructo general de Calidad de Vida incorporando las 14 categorías, pero solo cuatro fueron adecuadas. A continuación, se presenta el modelo y sus resultados (Figura 1).

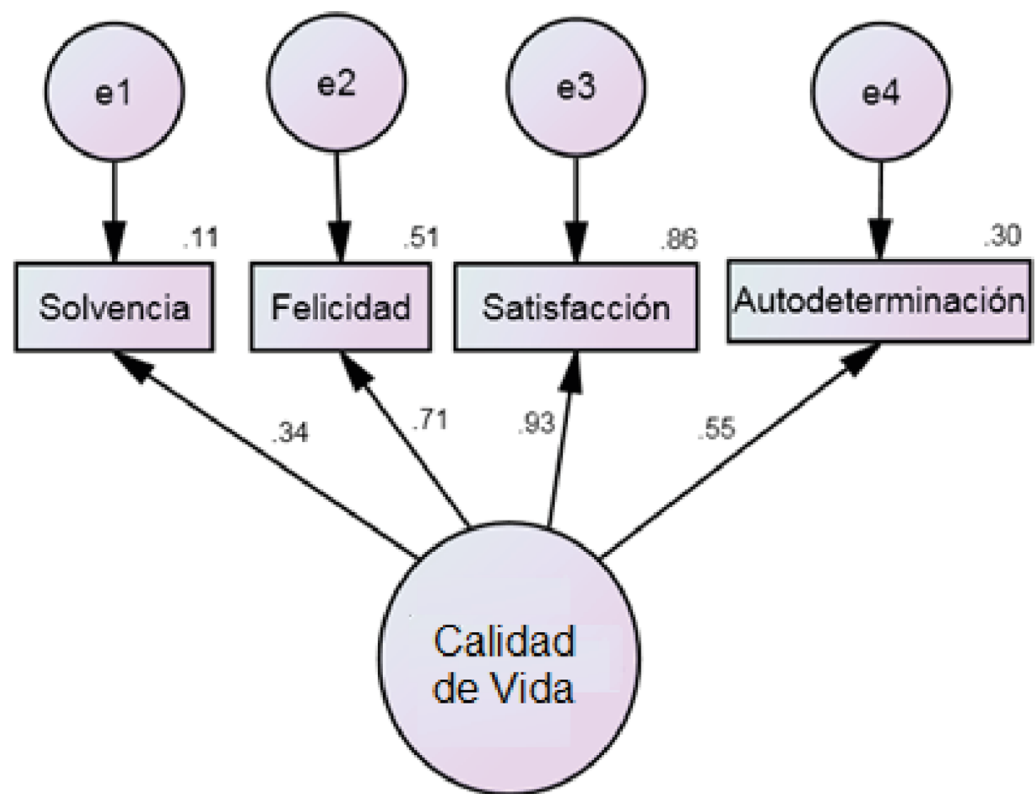

Figura 1. Modelo de medida con ajuste aceptable para el constructo de bienestar de segundo orden

Nota: $\mathrm{Ch}^{2}=8,818 \mathrm{p}=0,017, \mathrm{NFI}=0,999 ; \mathrm{RFI}=0,998 ; \mid \mathrm{Fl}=0,999 ; \mathrm{TLI}=0,998 ; \mathrm{CFI}=0,999 ; \mathrm{RMSEA}=0,017$

Se realizó el ajuste global del modelo resultando adecuadas las medidas de GFI y RMSR, RMSEA, indicando todas ellas que el modelo de Calidad de Vida es aceptable, así como valores aceptables para el Índice de Ajuste Normado (NFI= 0,999), Índice de Tucker-Lewis ( $T L I=0,998)$, los cuales tienen un umbral mayor al 0,90 deseado (Hair, Anderson, Tatham \& Black, 2010) lo que confirma la aceptabilidad del modelo. Los valores de medida de parsimonia, Índice de 
Ajuste Comparativo ( $\mathrm{CFI}=0,999)$, el Índice de Ajuste Incremental (IFI=0,999) también presentan valores al umbral deseado de 0,90 . Los pesos de $r$ para cada uno de los factores integrantes del constructo se presentaron para Solvencia $r=0,34$, Felicidad, $r=0,71$, Satisfacción $r=0,93$ y Autodeterminación $r=0,55$, los cuales en ciencias sociales se presentan como aceptables a partir de $r$ iguales o mayores de 0,30 . Todos estos valores en su conjunto ofrecen aceptabilidad del modelo Calidad de Vida.

Para evaluar la pertinencia de una relación entre ambos constructos (Calidad de vida y Sociocultural) se realizó el ajuste global del modelo (Figura 2). Como resultado se obtuvieron adecuadas las medidas de GFI y RMSR, RMSEA, así como valores aceptables para el Índice de Ajuste Normado (NFI=0,981, Índice de Tucker-Lewis ( $T L I=0,966)$, los cuales tienen un umbral mayor al 0,90 deseado. Por otro lado, la aceptabilidad del modelo se confirma con el Índice de Ajuste Comparativo ( $\mathrm{CFI}=0,982)$ y el Índice de Ajuste Incremental (IFI= 0,982 ). Los pesos de $r$ para cada uno de los factores fueron igual o mayores al 0,30 aceptable, así como un valor de $r=0,33$ entres ambos constructos (Bienestar Percibido y Sociocultural).

Dado que la variable solvencia en conjunto con las variables Satisfacción, Autodeterminación y Felicidad podían considerarse como agrupadas en un constructo Calidad de Vida (Figura 1), bajo este nuevo análisis (Figura 2) no tenía sentido llamarle así y se le renombró como variable latente Bienestar Percibido dado que las variables son más de índole personal.

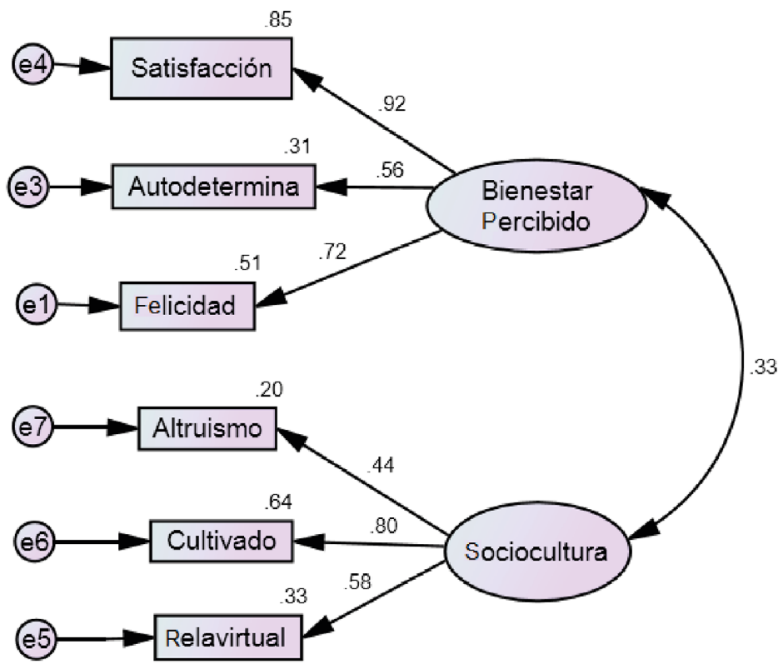

Figura 2. Modelo de dos variables latentes que se identifican con variables empíricas 
Con el fin de obtener el máximo nivel de explicación de la escala BIARE se realizaron análisis de trayectorias que pudiesen encontrar relaciones entre constructos. En el análisis de trayectorias (Figura 3) se encontraron valores de Índice de Ajuste Normado (NFI) de 0,994, Índice de Ajuste comparativo (CFI) de 0,944, Índice de Ajuste Relativo (RFI) de 0,963, Índice Tucker-Lewis (TLI) de 0,964 , todos ellos con valor mayor a 0,90. Asimismo, un valor del indicador Error de Aproximación Cuadrático Medio (RMSEA) de 0,077, el cual es adecuado por ser menor del 0.08 esperado. Las relaciones con carácter causal indican que el constructo Autodeterminación se relaciona con Satisfacción $(r=0,48)$ y con Solvencia $(r=0,16)$; Solvencia se relaciona con Satisfacción $(r=23)$ y Felicidad $(r=0,04)$. Destaca principalmente que Satisfacción a su vez se relaciona de manera importante con Felicidad $(r=0,65)$.

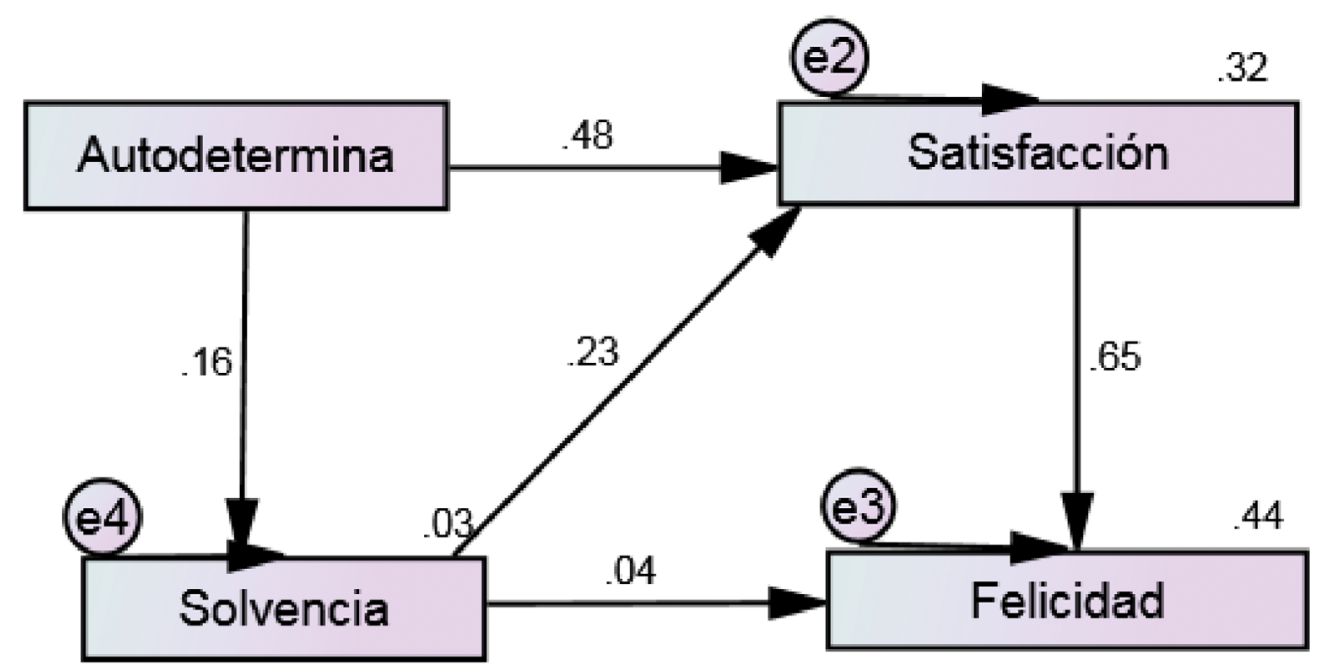

Figura 3. Modelo de trayectorias para las variables latentes que se identifican con variables empíricas del constructo calidad de vida.

Nota: $\mathrm{Chi}^{2}=64,954 \mathrm{p}=0,00, \mathrm{NFI}=0,994, \mathrm{RFI}=0,963, \mathrm{IFI}=0,994, \mathrm{TLI}=0,964, \mathrm{CFI}=0,994, \mathrm{RMSEA}=0,077$

Cabe desatacar que en los tres modelos evaluados los resultados no registraron varianzas negativas ni valores de correlación mayores a 1, esto sugiere la adecuación del cálculo de los modelos. Los tres modelos revisados lograron índices de ajuste satisfactorios de acuerdo a los criterios indicados por Kline (2015) y que señalan lo siguiente: (a) Root Mean Square error of approximation

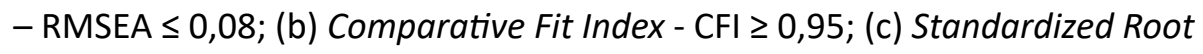
Mean Square Residual - SRMR $\leq 0,08$ ). Aún y cuando presenta limitaciones para reflejar el ajuste de un modelo se observó la Razón chi cuadrado $\left(x^{2} / g l\right)$ cuyo valor debe ser $\leq 3$ (Ruiz, Pardo \& San Martin, 2010) para completar la inspección de resultados. 
Al revisar los resultados de los modelos 1 y 2 , se presenta como tentativa la existencia de un constructo, que al intentar ser general posibilita la presencia de dos posibles factores subyacentes.

\section{DISCUSIÓN}

\subsection{La encuesta BIARE y su relación con estudios de bienestar subjetivo.}

Es importante destacar que en el estudio del bienestar subjetivo predominan las aproximaciones teóricas basadas en la estructura tripartita del bienestar subjetivo que se generó en los años ochenta, el cual implica un componente cognitivo de la satisfacción y los dos componentes afectivos, positivos y negativos (Busseri \& Sadava, 2011); no obstante, recientemente Diener Lucas \& Scollon (2009) plantearon una estructura conceptual jerárquica con cuatro componentes específicos. Los autores explicaron que en el nivel más alto de la jerarquía está el bienestar subjetivo, el cual se divide en los componentes: 1) afectos positivos, 2) afectos negativos, 3 ) juicios globales de la vida y 4) dominios específicos. Estos a su vez se subdividen en atributos tales como disfrute, felicidad y amor (primer componente); tristeza, enojo y preocupación (segundo componente); logros, significados y éxitos (tercer componente); pareja, trabajo, salud y recreación (cuarto componente). La organización planteada por Diener, Lucas \& Scollon (2009) se encuentra más próxima a un mapa conceptual que a un modelo empírico de los elementos de un constructo; no obstante lo descriptivo del planteamiento, autores icónicos del área de calidad de vida lo han tomado como punto de partida.

Ese planteamiento de cuatro componentes de Diener, Lucas \& Scollon (2009), solvencia, autodeterminación, felicidad en la presencia de afectos positivos, negativos y una división de satisfacción con la vida es compatible con el presente análisis.

\subsection{Sobre las escalas implicadas en BIARE}

El cuestionario BIARE arroja información valiosa sobre las condiciones de bienestar de la población mexicana, pero puede fortalecerse diseñando escalas con mejores posibilidades de ser comparadas a través de una correspondencia unívoca entre los números (misma distancia aritmética) de los atributos que se miden. Dicha disparidad entre escalas puede influir para que las respuestas de las personas y la correspondencia entre ellas, afecten los criterios de validez en el sentido convergente y discriminante entre variables. Por ejemplo, en las escalas que miden Felicidad (Gr-Feliz) es de 1-4 y la de 
Satisfacción (ENCSAT) de 0-10 puntos. Una sugerencia sería que ambas escalas fueran diseñadas con una correspondencia de 10 puntos.

\subsection{Sobre las variables implicadas en BIARE.}

En términos generales la información que se puede obtener del cuestionario BIARE es relevante respecto a la percepción que tienen los ciudadanos sobre sí mismos y de su entorno inmediato, lo cual es necesario y adecuado de medir, pero no contempla la percepción que tienen los mexicanos de su satisfacción respecto a variables macro-sistémicas y la influencia que estas tienen sobre sus vidas, como son: participación ciudadana en el desarrollo, economía, democracia, libertad de expresión y rendición de cuentas. Es decir, BIARE en su versión 2012 no presenta un enfoque multidimensional del bienestar. Esta observación es relevante porque se ha documentado en diferentes encuestas que los mexicanos reportan altos niveles de satisfacción con la vida en términos personales (Helliwell, Layard \& Sachs, 2012; The Organization for Economic Co-operation and Development [OECD], 2015; Vera, Velasco, Grubits \& Salazar, 2016). Pero en otros estudios que miden satisfacción en relación a variables que son meso o macro sociales se encuentra que no ocurre lo mismo, por ejemplo:

1. Los mexicanos reportan bajos niveles de satisfacción con el país en términos de democracia plena, con un $44 \%$ inferior a la media de 46 $\%$ para la región de Latinoamérica. Asimismo, México es el país en Latinoamérica con el valor más alto obtenido cuando se les pregunta si es posible la democracia sin partidos políticos (con 45 puntos de aprobación, en relación a una media de 31) y sin congreso (38) en relación a una media de 27 (Latinobarómetro, 2013). Este último aspecto es relevante porque coincide con el repudio generalizado a través de redes sociales hacia la clase política en relación con la corrupción y legitimidad de las elecciones, lo cual hace percibirse entre los mexicanos que la democracia en México es endeble o nula cuando se trata de evaluar al sistema político (Tenorio, 2015).

2. Otra encuesta arroja información que indica que la satisfacción laboral que reportan los mexicanos es la más baja respecto a los países de América Latina, $12 \%$ de los trabajadores se reportan satisfechos, $60 \%$ desconectados (tienen objeciones o reportan clima laboral desfavorable) y un $28 \%$ repudia totalmente su trabajo (Gallup, 2013).

3. Sumado a este panorama laboral el salario mínimo de los mexicanos durante 25 años ha perdido el $78,71 \%$ de su poder adquisitivo, lo cual implica que se necesitan por lo menos tres salarios mínimos para comprar 
la canasta básica (Centro de Análisis Multidisciplinario [CAM], 2015). En cambio, la jornada laboral en México, a partir de 2009, ha sido una de las de mayor promedio de horas dedicadas al trabajo (la más alta reportada con 2,237 en el 2013) dentro de los países miembros de la Organización para la Cooperación y el Desarrollo Económicos (The Organization for Economic Co-operation and Development [OECD], 2014).

Bajo un contexto estrictamente económico cabe señalar que los programas de reducción de la pobreza en México han fracasado porque estos no han reducido significativamente el número de pobres en el país, las cifras del Consejo Nacional de Evaluación de la Política de Desarrollo Social (Consejo Nacional de Evaluación de la Política de Desarrollo Social [CONEVAL], 2015) muestran que: la pobreza extrema en porcentaje de la población se incrementó en Colima (60\%), Nayarit (43\%) y Nuevo León (33 \%). Así también, a nivel estatal, entre 2010 y 2012, la pobreza extrema por número de personas aumentó en Puebla (57 mil), Jalisco (54 mil), Michoacán (52 mil), Nayarit (46 mil) y Quintana Roo (35 mil).

Así pues, factores como los anteriores no los contempla BIARE al evaluar el bienestar y satisfacción de los mexicanos; para obtener una mejor explicación de sus condiciones. En la encuesta BIARE se tendría que preguntar a las personas cómo perciben su nivel de felicidad o satisfacción respecto a su ingreso, educación, seguridad, país, gobierno y libertad, por ejemplo. Al parecer los indicadores actuales de BIARE se relacionan más con la felicidad intrínseca o cultural de los mexicanos ¿Cómo es posible que los mexicanos con niveles de inseguridad política, económica y personal seamos tan o más felices que países considerados de primer mundo? Los estudios de Díaz-Guerrero (2003) han ilustrado por décadas el poder que tiene la cohesión afiliativa tan arraigada del mexicano hacia dentro de la familia (implosión) y de cómo esta erige los valores y afectos en su convivencia más que los atributos individuales que cada persona pudiese tener. Es decir, los niveles tan altos de bienestar que reportan a nivel mundial podrían no estar representando su bienestar global como nación, y más bien estos pueden deberse a un sesgo social que deriva de percibir a la familia como el "recipiente" o la razón de la felicidad.

En un análisis anterior (Vera, Velasco, Grubits \& Salazar, 2016) en los que se compararon las condiciones de vida de las personas con niveles extremos de felicidad en México se encontró que cuando el tejido familiar está roto, e incluso en ella se genera violencia hacia las personas, los niveles de felicidad y satisfacción de los mexicanos se desploman a niveles mínimos o nulos. 
Esto parece indicar que el reducto más importante para el bienestar de la población mexicana es la familia y si esta se fracciona, los mexicanos reportan los niveles más paupérrimos de bienestar subjetivo.

Entonces, tal como se encuentra la estructura de la encuesta de BIARE, no se permite evaluar con precisión la contribución de factores meso y macro-sociales en la percepción y bienestar de los mexicanos, ni se les pude comparar con otros países; lo que se está midiendo con BIARE son factores más inmediatos del contexto (micro-sistema). Por esta razón es necesario que en BIARE se integre un modelo multidimensional de bienestar para los mexicanos (Stiglitz, Sen \& Fitoussi, 2010), el cual pueda ofrecer un mayor poder explicativo. Se requiere un modelo robusto, que incluya necesariamente variables micro, meso y macro-sociales. Asimismo, estas con múltiples niveles o dominios de interacción entre subsistemas deberían medirse en una misma muestra representativa de la sociedad mexicana para que la información tenga validez convergente y discriminante, lo cual permitiría describir y explicar tipologías o características más precisas de los ciudadanos y su comportamiento en términos sistémicos.

Así también, un enfoque teórico y sistémico se hace necesario porque los problemas y soluciones sociales, por definición, tienen un carácter sistémico (son y se comportan como sistemas). Así pues, cualquier solución o explicación que se quiera hacer de los individuos comportándose en la sociedad debe incluir un enfoque sistémico que permita saber "quiénes necesitan qué, cómo se satisfacen tales necesidades y qué deberes tenemos con los necesitados" (Bunge, 2008, p.24). Además, se debe preguntar a los ciudadanos sobre cómo piensan contribuir a su bienestar y cómo ellos se sienten responsables de sus condiciones de vida.

\subsection{Sobre modelación}

A través de la modelación se pudo identificar que la encuesta BIARE 2012 tiene valor explicativo en relación a variables de atribución individual, y está conformada principalmente por dos variables:

Bienestar percibido. a) Satisfacción con la vida que ha tenido el individuo (sin referentes ambientales fuera de la familia), b) Autodeterminación, cómo contribuye el individuo a su bienestar, y c) Felicidad, qué tan feliz es con su vida. Todas ellas dependen de una valoración del individuo hacia sí mismo. 
Variable latente socio-cultural. Está relacionada con las siguientes variables empíricas: a) Altruismo, que tanto contribuyó o ayudó a los demás, familia, amigos y desconocidos, b) Cultivado, en términos de si el individuo realiza actividades educativas o culturales,, y c) Relaciones virtuales, que refieren todos aquellos vínculos que tiene el individuo con familiares y amigos a través de redes de comunicación y sociales.

La encuesta BIARE 2012 no confronta a los mexicanos y sus familias con variables que están en el plano del país como son criminalidad, acceso a la educación, economía, equidad, satisfacción con el gobierno, etc., sino que se le cuestiona estrictamente en relación al contexto familiar o individual psicológico. La encuesta BIARE -como medida de bienestar de los mexicanosdebe definirse como multidimensional, en la cual deben considerarse las siguientes dimensiones: 1) estándares de la vida material (ingresos, consumo y riqueza), 2) salud, 3) educación, 4) actividades personales (incluyendo al trabajo), 5) voz política y gobernabilidad, 6) conexiones y relaciones sociales, 7) medioambiente (condiciones actuales y futuras) y 8) Inseguridad (económica, física), tal como se considera en países como Alemania (Peichl \& Pestel, 2013).

La encuesta BIARE 2012 se presentó como una prueba de pilotaje o de interés "experimental" (INEGI, 2012). Obedeciendo a este primer acercamiento de carácter "experimental", la encuesta BIARE sigue en constante cambio. En la versión BIARE que se aplicó en el 2015, llamada versión ampliada (INEGI, 2014), presenta nuevas adecuaciones, lo cual presenta retos para los investigadores en la evaluación de sus componentes y de su pertinencia para medir bienestar de los mexicanos. La presente investigación tuvo como objetivo explorar la estructura y relación que tienen los módulos en la encuesta BIARE aplicada en el año 2012 mediante el modelamiento estructural (AMOS). El análisis tiene limitaciones en el sentido de que las oportunidades detectadas en esta versión pueden no coincidir con los tiempos e intereses gubernamentales que originan que se cambien sus componentes y/o variables en cada versión de BIARE. No obstante, creemos que es pertinente seguir contribuyendo en el análisis y discusión de las propiedades que tienen los módulos de BIARE para medir bienestar.

En resumen, en orden de complejidad, para mejorar la encuesta BIARE y conocer las condiciones de bienestar de los mexicanos se requiere:

1. Homologar las escalas que miden atributos similares o compatibles. 
2. Incluir variables relevantes de orden multidimensional (micro, meso y macro-social).

3. De cumplirse los dos primeros criterios un modelo multidimensional pudiese ser probado mediante modelación con ecuaciones estructurales para dar coherencia al orden de los componentes y variables implicadas en las condiciones de vida de los mexicanos.

Financiamiento: Proyecto Etnopsicologia y calidad de vida (60148). Coordinación de Desarrollo Regional. Centro de Investigación en Alimentación y Desarrollo A.C.

Agradecimientos: Al Instituto Nacional de Geografía e Informática por el acceso a las bases de datos y al Departamento de Psicología y Ciencias de la Comunicación de la Universidad de Sonora por el apoyo documental.

\section{REFERENCIAS}

Allais, M. \& Hagen, O. (1979). Expected utility hypothesis and the Allais paradox,; The Netherlands. Reidel, Dordrecht

Bunge, M. (2008). Filosofía y Sociedad. México: Siglo XXI.

Busseri, M. A., \& Sadava, S. W. (2011). A review of the tripartite structure of subjective well-being: Implications for conceptualization, operationalization, analysis, and synthesis. Personality and Social Psychology Review, 15(3), 290-314. https://doi. org/10.1177/1088868310391271

Centro de Análisis Multidisciplinario [CAM], (2015). Reporte de Investigación 120. México: Esclavitud moderna. Cae 78,71 \% el poder adquisitivo. Centro de Análisis Multidisciplinario. Universidad Nacional Autónoma de México. Recuperado de: http://cam.economia.unam.mx/reporte-de-investigacion-120-mexico-esclavitud-moderna-cae-78-71-el-poder-adquisitivo/

Cardona, D. \& Agudelo, H. B. (2005). Construcción cultural del concepto calidad de vida. Rev. Fac. Nac. Salud Pública, 23(1), 79-90. Recuperado de http:// www.udea.edu.co/wps/wcm/connect/udea/3c569269-fd71-4c59-9e190e241ef0b1e3/2005_Construcci\%C3\%B3n+cultural+del+concepto+calidad+de+vida.pdf?MOD=AJPERES

Consejo Nacional de Evaluación de la Política de Desarrollo Social [CONEVAL], (2015). Consideraciones para el proceso presupuestario 2016. Consejo Nacional de Evaluación de la Política de Desarrollo Social. México. Recuperado de: http:// www.coneval.gob.mx/Informes/Evaluacion/Consideraciones_presupuestales/ Consideraciones_presupuestales_2016.pdf

Cummins, R.A. (2005). Moving from the quality of life concept to a theory. Journal of Intellectual Disability Research, 49(10), 699-706. DOI: https://doi.org/10.1111/ j.1365-2788.2005.00738.x

Díaz-Guerrero, R. (2003). Bajo las garras de la cultura. Psicología del mexicano 2. México, D.F: Trillas.

Diener, E. (2000). Subjective well-being: The science of happiness and a proposal for a national index. American psychologist, 55(1), 34. http://dx.doi. org/10.1037/0003-066X.55.1.34 
Diener, E., Lucas, R. E. \& Scollon, C. N. (2009). Beyond the hedonic treadmill: Revising the adaptation theory of well-being. Ledwig, P. (Ed.) In The science of well-being (pp.103-118). The Netherlands. Springer.

Dow, G.K. \& Juster, F.T. (1985). Goods, time and well-being: the joint dependency problem, in F.T. Juster and F.P. Stafford (eds.). Time, goods and well-being. Ann Arbor, Michigan: Institute of Social Research.

Gallup (2013). Worldwide, 13 \% of Employees are Engaged at Work (by Steve Crabtree). Recuperado de: http://www.gallup.com/poll/165269/worldwide-employees-engaged-work.aspx.

Hair, J., Anderson, R., Tatham, R. \& Black, W. (2010). Multivariate data analysis (7a ed.). Upper Saddle River, New York: Prentice-Hall.

Helliwell, J., Layard, R. \& J. Sachs, J., Eds. (2012). World happiness report. Earth Institute. Recuperado de: http://www.earth.columbia.edu/articles/view/2960.

Hoorn, A. (2007). A Short introduction to subjective well - being its measurement, correlates and policy uses. Nijmegen Center for Economics (NICE), Radboud University Nijmegen. https://www.oecd.org/site/worldforum06/38331839.pdf

Instituto Nacional de Estadística y Geografía [INEGI], (2012). Presenta el INEGI cifras sobre el bienestar subjetivo de los mexicanos. Boletín de Prensa, 431(12), 1-3. Recuperado de http://www.miguelcarbonell.com/artman/uploads/1/bienestar_ subjetivo.pdf.

Instituto Nacional de Estadística y Geografía [INEGI], (2014). Módulo de Bienestar Autorreportado. México. Instituto Nacional de Estadística y Geografía e informática Recuperado de http://www.beta.inegi.org.mx/contenidos/proyectos/ investigacion/bienestar/ampliado/doc/biare_ampliado_cuest.pdf

Kahneman, D, Knetsch, J. \& Thaler, R. (1991). The endowment effect, loss aversion, and status quo bias. Journal of Economic Perspectives, 5, 193-206. https://doi. org/10.1257/jep.5.1.193

Kahneman, D. \& Tversky, A. (1979). Prospect theory: an analysis of decision under risk. Econometrica, 47(2), 263-291. https://doi.org/10.2307/1914185

Kahneman, D, Wakker, P.P. \& Sarin, R. (1997). Back to Bentham? Explorations of experienced utility, Quarterly Journal of Economics, 112, 375-405. https://doi. org/10.1162/003355397555235

Kline, R. B. (2015). Principles and practice of structural equation modeling. New York: Guilford publications.

Latinobarómetro (2013). Informe 2013. Santiago de Chile: Corporación Latino barómetro. Recuperado: http://www.latinobarometro.org/documentos/LATBD_ INFORME_LB_2013.pdf.

McMillan J. y Schumacher S. (2008) Investigación Educativa. Quinta Edición. México: Pearson, pp.129-177.

The Organization for Economic Co-operation and Development [OECD], (2014). OECD Factbook 2014: Economic, Environmental and Social Statistics. Paris: OECD Publishing. http://dx.doi.org/10.1787/factbook-2014-en

The Organization for Economic Co-operation and Development [OECD], (2015). Measuring Well-being in Mexican States. Paris: OECD Publishing. http://dx.doi. org/10.1787/97892642466072-en

Peichl, A. \& Pestel, N. (2013). Multidimensional Well-Being at the Top: Evidence for Germany. Fiscal Studies, 34(3), 355-371. https://doi.org/10.1111/j.14755890.2013.12010.x 
Rojas, M. \& Jiménez, E. (2008). Pobreza subjetiva en México: el papel de las Normas de evaluación del ingreso. Perfiles Latinoamericanos, (032), 11-33. Recuperado de http://perfilesla.flacso.edu.mx/index.php/perfilesla/article/view/173/117

Ruiz, M. A., Pardo, A. \& San Martín, R. (2010). Modelos de ecuaciones estructurales. Papeles del psicólogo, 31(1), 34-45. http://www.papelesdelpsicologo.es/ pdf/1794.pdf

Stiglitz, J. E., Sen, A. \& Fitoussi, J. P. (2010). Report by the commission on the measurement of economic performance and social progress. Paris: Commission on the Measurement of Economic Performance and Social Progress. Recuperado de http://ec.europa.eu/eurostat/documents/118025/118123/Fitoussi+Commission+reporthttp://ec.europa.eu/eurostat/documents/118025/118123/Fitoussi+Commission+report

Tenorio, D. (2015). Las redes sociales al rescate. Foreign Affairs. Recuperado de http://revistafal.com/las-redes-sociales-al-rescate/

Van Praag, B. M. \& Frijters, P. (1999). 21 The measurement of welfare and well-being: The Leyden approach. En E. Diener, D. Kahneman, \& N. Schwarz (Eds.), Well-being: the foundations of hedonic psychology (pp.413-433). New York: Russel Sage Foundation. Recuperado de https://pure.uva.nl/ws/files/3148285/8399_The_ measurement_of_welfare_and_well_being_The_Leyden_Approach_Praag_.pdf

Van Praag, B.M.S. (1968). Individual welfare functions and consumer behavior. A theory of rational irrationality. (Ph.D.-thesis) Amsterdam. North-Holland Publishing Company.

Van Praag, B.M.S. (1971). The welfare function of income in Belgium: an empirical investigation. European Economic Review, 2(3), 337-369. https://doi. org/10.1016/0014-2921(71)90045-6

Veenhoven, R. (1996). Happy life-expectancy: a comprehensive measure of quality of life in nations. Social Indicators Research, 39, 1-58. https://doi.org/10.1007/ BF00300831

Vera, J.A., Velasco, F.J., Grubits, H.B. \& Salazar, I.G. (2016). Parámetros de inclusión y exclusión en los extremos de la felicidad y satisfacción con la vida en México. Acta de Investigación Psicológica. 6, 2395-2403. http://dx.doi.org/10.1016/j. aipprr.2016.06.002 